\title{
HIGH-POWER LASER WELDING OF AUSTENITIC STAINLESS STEEL WITH ELECTROMAGNETIC CONTROL OF WELD POOL
}

\author{
M. BACHMANN, V. AVILOV, A. GUMENYUK and M. RETHMEIER \\ BAM - Federal Institute for Materials Research and Testing \\ 87 Unter den Eichen, 12205, Berlin, Germany. E-mail: marcel.bachmann@bam.de
}

\begin{abstract}
Laser deep-penetration welding became a widely applied tool in industrial applications due to available laser power of $20 \mathrm{~kW}$ and more for the single-pass welding of steel plates of up to $20 \mathrm{~mm}$ thikness. Above a critical limit, liquid metal tends to drop out of the bead due to hydrostatic pressure. Laser welding, in contrast to electron beam welding technique, allows for an electromagnetic manipulation of fluid flow in the weld pool. AC electromagnetic system for compensation of the hydrostatic pressure by induced Lorentz forces in the melt was experimentally and numerically investigated for single-pass full-penetration welding of up to $20 \mathrm{~mm}$ thikness austenitic stainless steel plates of grade AISI 304. It was shown that the application of 200-234 mT magnetic fields at oscillation frequency of around $2.6 \mathrm{kHz}$ lead to a full compensation of hydrostatic forces in the melt for plate 10-20 mm thick, respectively. Coupled fluid flow, thermal and electromagnetic finite element simulations were done with different applied magnetic flux densities and oscillation frequencies calculating for the optimal magnetic field strength to avoid melt sagging in the weld pool. The simulation results point to a lower magnetic field density needed for that purpose. The reason for that can lie in the magnetic properties of the material not being totally non-ferromagnetic. 17 Ref., 1 Table, 5 Figures.
\end{abstract}

Keywords: laser welding, high power, austenitic stainless steels, drop out of bead, control magnetic field, hydrostatic force compensation, modeling of fluid flow, calculation

In the course of the last decade, the availability of laser sources within the power class above $10 \mathrm{~kW}$ made it possible to weld ever thicker aluminium and steel plates of up to $30 \mathrm{~mm}$ in a full-penetration process $[1,2]$. Such a process has the advantage of being very efficient along with the well-known key benefits of laser welding compared to multipass arc welding processes, e.g. the low heat input, high welding speeds as well as low distortion [3].

The present investigation deals with the single-pass laser welding of up to $20 \mathrm{~mm}$ thickness stainless steel plates of AISI 304 grade. The conventional method to weld thick components is to use EBW [4, 5], which brings up challenges for large modules due to the need of technical vacuum. Nowadays, modern laser beam sources enable a stable single-pass welding process up to $16 \mathrm{~mm}$ penetration for steel $[6,7]$.

Above a critical limit, the surface tension of the molten material cannot balance the hydro-

\footnotetext{
*Basing on the paper presented at the Int. Conf. on Laser Technologies in Welding and Materials Processing (27-31 May 2013, Katsively, Crimea, Ukraine).

(C) M. BACHMANN, V. AVILOV, A. GUMENYUK and M. RETHMEIER, 2014
}

static pressure of the melt and drops out during the welding before solidification occurs.

Another challenge are the highly dynamical processes in the welding zone, e.g. due to Marangoni flow and natural convection.

Laser welding in contrast to EBW allows for an electromagnetic treatment of the melt. Electromagnetic technologies in the processing of metals are widespread and range from crystal growth and cold crucible melting to the porosity prevention and surface treatment [8], and also stirring [9] in welding applications.

The approach in this investigation is the application of oscillating magnetic field perpendicular to welding direction below the welding zone, which induces eddy currents contactless. The resulting volumetric Lorentz forces in the melt counteract the effect of gravitational forces and compensate for the hydrostatic pressure. System of electromagnetic weld pool control was already experimentally [10,11] investigated for steel of up to $18 \mathrm{~mm}$ thickness and aluminium alloys $30 \mathrm{~mm}$ thick. Numerical justification for $20 \mathrm{~mm}$ aluminium was presented in [12].

The present investigation deals with the numerical calculation and experimental validation of the electromagnetic weld support system for $20 \mathrm{~mm}$ stainless steel AISI 304. Representative simulation studies of a fluid flow simulation coupled with an electromagnetic processing are presented in [12-14]. 

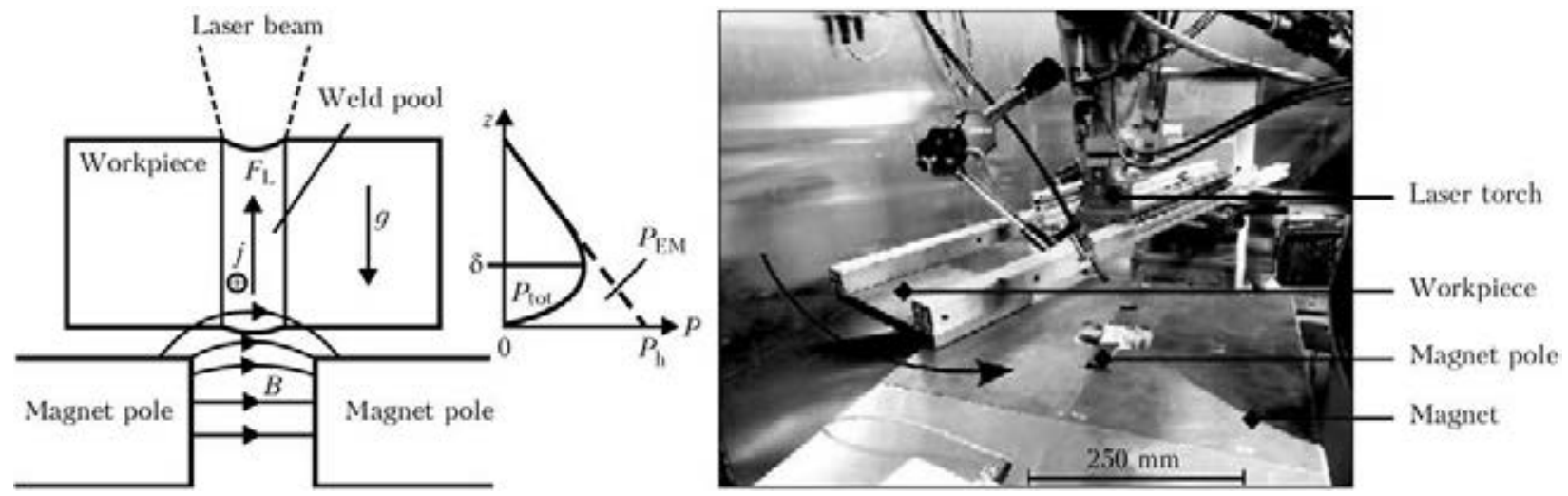

Figure 1. Scheme of the electromagnetic weld pool support system $(a)$ and experimental setup $(b)$

Experimental setup. Working principle of the applied electromagnetic weld pool support is sketched in Figure 1, $a$. The oscillating magnetic field $B$ is located in the centre below the weld pool and induces eddy currents $j$ within the skin depth $\delta=(\pi f \mu \sigma)^{-1 / 2}$ of the material, where $f$ is the oscillation frequency; $\mu$ is the magnetic permeability, and $\sigma$ the electric conductivity. Interaction of the induced currents with applied magnetic field produces a Lorentz force $F_{\mathrm{L}}=j B$ in the melt, which counteracts the hydrostatic pressure and, in the case of an optimal control, ensures balancing of pressures on the upper and lower weld surfaces to avoid dropping of the melt.

The magnet was located $2 \mathrm{~mm}$ below the workpiece. The magnet poles had a distance of $25 \mathrm{~mm}$, and their cross section had the dimensions $25 \times$ $\times 25 \mathrm{~mm}$. The experimental setup can be seen in Figure 1, $b$.

The bead-on-plate welds were done with fibre laser with beam power of up to $18 \mathrm{~kW}$. Welding of $20 \mathrm{~mm}$ AISI 304 steel produced no reasonable result as the liquid material was blown out of the weld. Therefore, the $20 \mathrm{~mm}$ joints were made on $10 \mathrm{~mm}$ AISI 304 austenitic steel at the root side and $10 \mathrm{~mm}$ S235 ferritic steel above as the higher surface tension of S235 steel stabilizes the

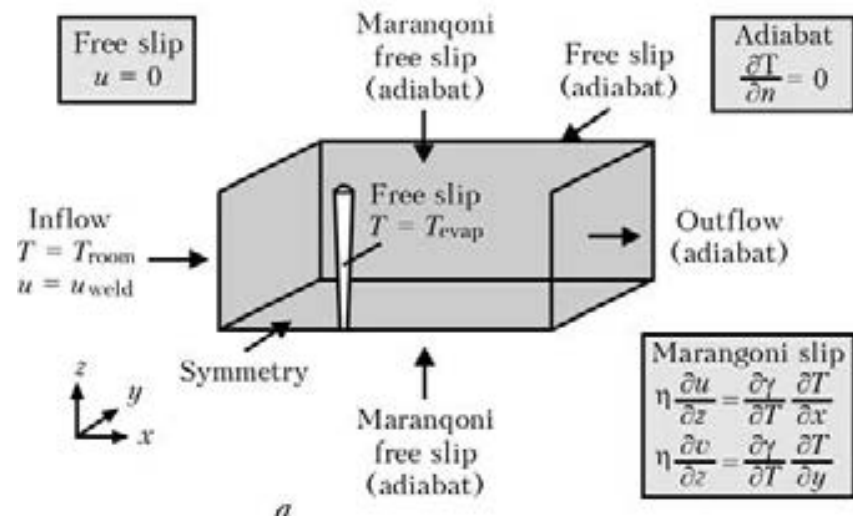

weld surface. The penetration depth of magnetic field was adjusted at around $10 \mathrm{~mm}$ so that the magnetic characteristics of the ferritic S235 steel do not influence the applied magnetic field significantly. The $20 \mathrm{~mm}$ case was supposed to show the principal applicability of the magnetic weld pool support for even higher plate thicknesses. The laser and optics properties are summarized below:

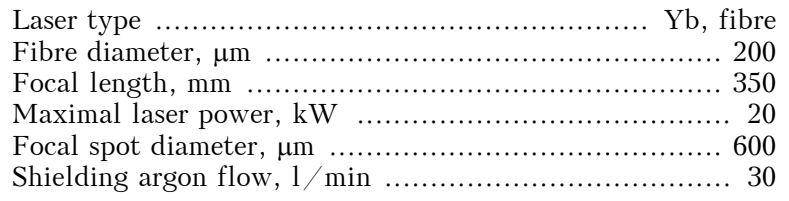

Mathematical modelling. The numerical model calculates the turbulent fluid flow equations, i.e. mass conservation with mass density $\rho$ and velocity $u$, and the Navier-Stokes equations with dynamic viscosity $\eta$, pressure $p$ and source term $F$ :

$$
\nabla(\rho u)=0
$$

$$
\rho(u \nabla) u=-\nabla p+\nabla\left[\eta\left(\nabla u+(\nabla u)^{T}\right)-\frac{2}{3} \eta(\nabla u) I\right]+F,
$$

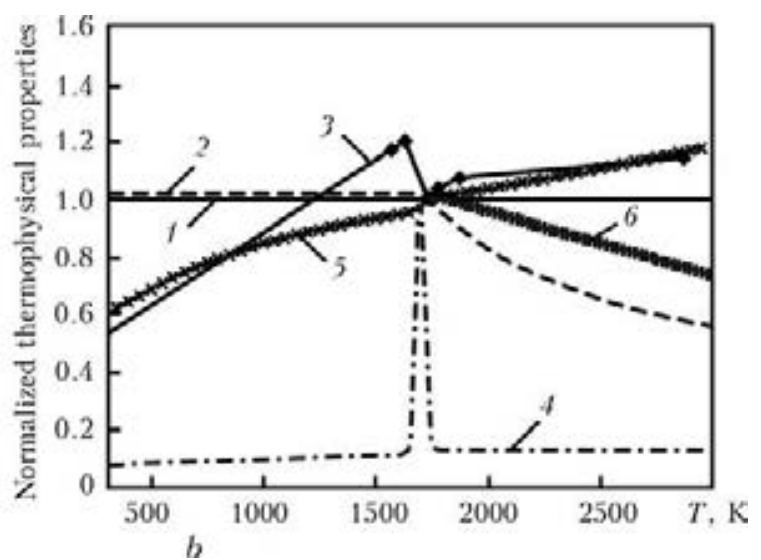

Figure 2. Boundary conditions $(a)$, and thermophysical properties of stainless steel AISI 304 at $T_{\text {melt }}(b): 1-$ density $\rho / \rho ; 2-$ dynamic visvosity $\eta / \eta ; 3-$ heat conductivity $\lambda / \lambda ; 4-$ heat capacity $C_{p}^{\text {eff }} / C_{p}^{\text {eff }} ; 5-$ electrical resistivity $\rho_{\mathrm{el}} / \rho_{\mathrm{el}} ; 6-$ surface tension $\gamma / \gamma$ 


$$
F=-\rho g-c_{1} \frac{\left(1-f_{1}\right)^{2}}{f_{1}^{3}+\varepsilon}\left(u-u_{\text {weld }}\right)+<j B>.
$$

In (3), the first term accounts for the gravitational influence, the second term refers to braking of the solidified material down to processing velocity, and the last term - to applied timeaverage of the Lorentz force; $f_{1}$ is the liquid fraction, and $c_{1}$ and $\varepsilon$ are the constants.

Additionally, the energy equation with effective heat capacity $C_{p}^{\text {eff }}$ accounting also for the latent heat of fusion, temperature $T$ and heat conductivity $\lambda$ is solved:

$$
p C_{p}^{\text {eff }} u \nabla T=\nabla(\lambda \nabla T) .
$$

The Maxwell equations with electric field $E$ accounts for the applied electromagnetic influence of the weld support system. The influence of flow field on electric current density distribution is described by the generalized Ohm's law:

$$
\nabla B=\mu j, \quad \nabla E=\frac{\partial B}{\partial t}, \quad j=\sigma(E+u B) .
$$
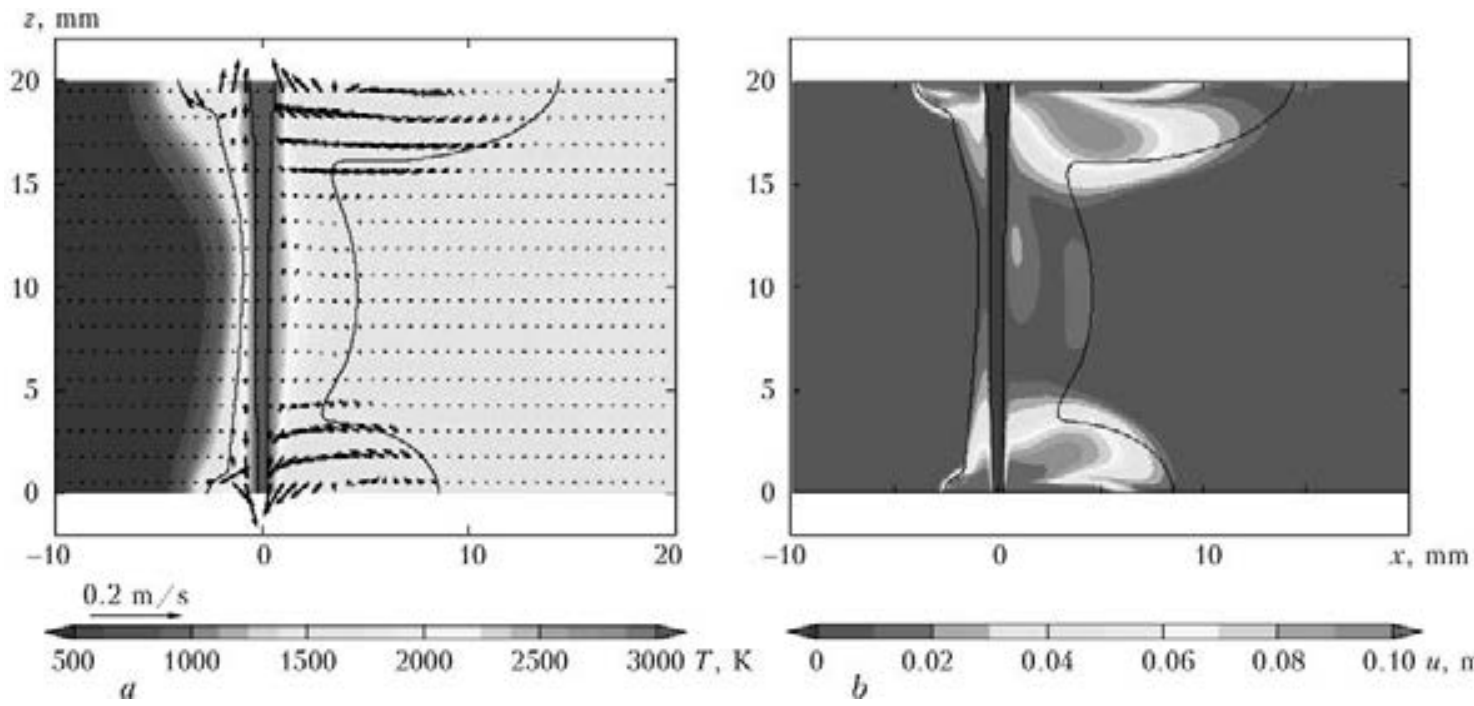

Figure 3. Symmetry plane of simulation results for case with optimal electromagnetic control of the hydrostatic pressure at welding speed of $0.4 \mathrm{~m} / \mathrm{min}: a-$ temperature distribution and velocity vectors; $b$ - velocity magnitude distribution
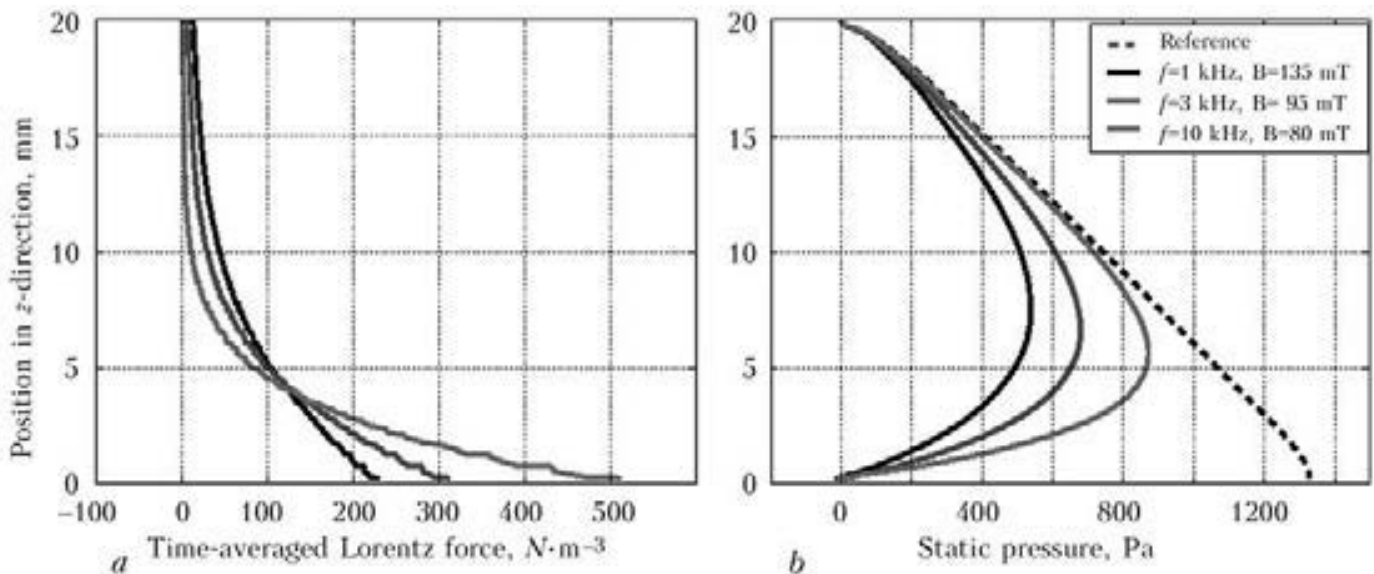

Figure 4. Pressure distribution $3 \mathrm{~mm}$ behind the keyhole in vertical axis $(a)$, and time-averaged vertical component of the Lorentz force $(b)$ 


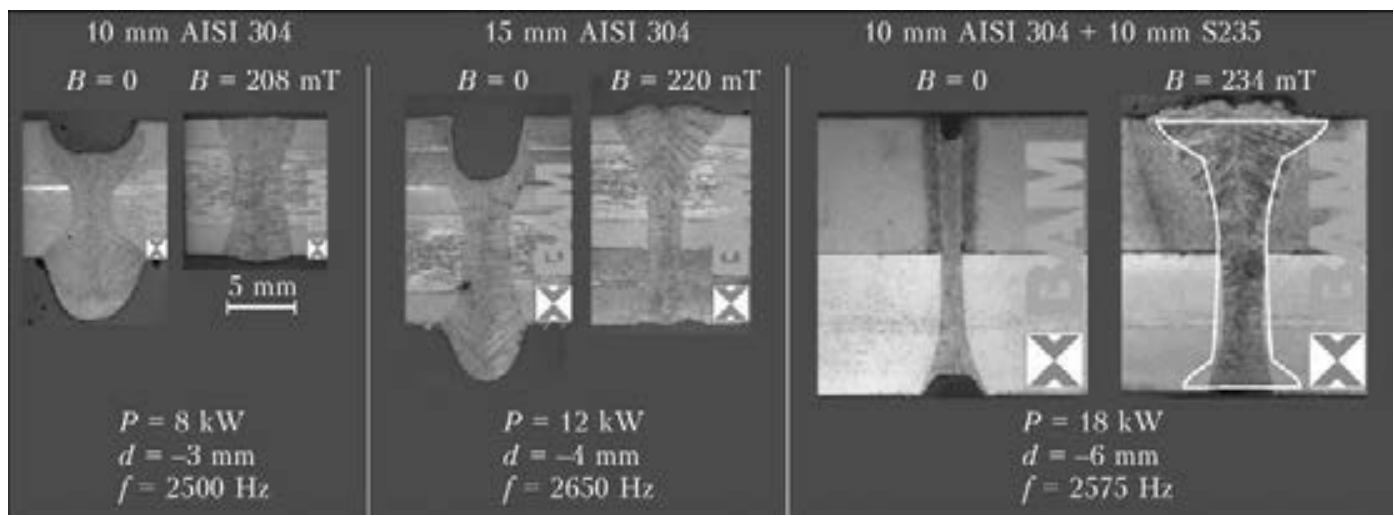

Figure 5. Single-pass laser welding without $(B=0)$ and with $(B \neq 0)$ optimal parameters of the electromagnetic support system for 10-20 mm thickness at $0.4 \mathrm{~m} / \mathrm{min}$ welding speed. Laser power $P$ and focus depth $d$ were adapted with respect to plate thickness; for $20 \mathrm{~mm}$ thickness the simulational cross section is overlayed at $95 \mathrm{mT}$ and $3 \mathrm{kHz}$

$3 \mathrm{kHz}$ that leads to penetration depth of the magnetic field of around $10 \mathrm{~mm}$ in the liquid phase of material. The peak $u$ values in the regions near the free surfaces of weld pool are due to the Marangoni flow directed from hot to cold regions as the surface tension increases along that path. Therefore, the bead is elongated at both surfaces. At the lower surface, this elongation is smaller due to presumed geometry of keyhole with smaller diameter at the lower side.

Figure 4, $a$ shows the hydrostatic pressure compensation in the weld $3 \mathrm{~mm}$ behind the keyhole in symmetry plane for three values of frequencies. It shows that pressure values at both surfaces are nearly equal, so that dropping of melt cannot occur due to gravity effects, and calculated pressure distribution corresponds well with vertical component of the Lorentz force (Figure 4,b).

Experimental results. The experimental results for thicknesses between $10 \mathrm{~mm}$ and $20 \mathrm{~mm}$ are shown in Figure 5. Up to $15 \mathrm{~mm}$, the material AISI 304 was used; for $20 \mathrm{~mm}$ - combination of steels AISI 304 (lower side) and S235 (upper side) was used, due to stability issues of welding process at the upper weld pool side and the higher surface tension of structural steel. Up to thickness of 15 $\mathrm{mm}$, the reference cases show severe sagging of material, whereas the case of $20 \mathrm{~mm}$ is associated with unstable welding process and material loss on root and top side. The magnetic flux density needed to avoid sagging increases slightly with higher material thicknesses, and a state of optimal compensation can be reached for any thickness. The cross sections of simulation with $B=95 \mathrm{mT}$ at $3 \mathrm{kHz}$ oscillation frequency corresponds well with experiment (234 $\mathrm{mT}$ and $2.6 \mathrm{kHz})$.

\section{Conclusion}

Electromagnetic weld pool support was successfully applied for up to $20 \mathrm{~mm}$ thickness stainless steel, and severe sagging of liquid material could be prevented. The simulations show smaller value of the magnetic flux density for compensation of hydrostatic pressure. Only slight increase of magnetic field in the experiments for different thicknesses allows for speculations about a further effect, that must be compensated for, e.g. other dynamic oscillatory processes in the melt associated with the vapor phase in keyhole and corresponding reaction forces or even the influence of weakly ferromagnetic properties of the material, especially in the light of the exact predictions for magnetic flux density for aluminium alloy AlMg3 [12].

Acknowledgements. Financial funding of the Deutsche Forschungsgemeinschaft DFG (Bonn, Germany) under Grant No. DFG GU $1211 / 2-1$ is gratefully acknowledged.

1. Avilov, V. et al. (2012) Sci. and Technol. of Weldind and Joining, 17, 128-133.

2. Vollertsen, F. et al. (2010) Welding in the World, 54, 62-70.

3. Ready, J.F. et al. (2001) LIA Handbook of Laser Materials Processing

4. Sanderson, A. et al. (2000) Fusion Eng. Des., 49/50, 77-87.

5. Kohyama, A. et al. (1984) J. Nucl. Mater., 122, $772-776$

6. Kawahito, Y. et al. (2009) Sci. and Technol. of Welding and Joining, 14, 288-294.

7. Shin, M. et al. (2010) Transact. of JWRI, 39, 33-38.

8. Schneider, A. et al. (2013) Phys. Proc., 41, 4-11.

9. Vollertsen, F. et al. (2006) J. Laser Appl., 18, 28-34.

10. Avilov, V.V. et al. (2009) Proc. of EPM (2009, Dresden, Germany)

11. Avilov, V.V. et al. (2012) Sci. and Technol. of Welding and Joining, 17, 128-133.

12. Bachmann, M. et al. (2012) J. Phys: Appl. Phys. D, 45, 13.

13. Gatzen, M. et al. (2009) Proc. of LAMP

14. Velde, O. et al. (2001) Int. J. Heat Mass Transfer, 44, 2751-2762.

15. Sahoo, P. et al. (1988) Metall. and Mat. Transact. $B, 19,483-491$

16. Mills, K.C. (2002) Recommended values of thermophysical properties for selected commercial alloys. Woodhead Publ.

17. Wilthan, B. et al. (2008) Int. J. Thermophys., 29, 434-444 